\title{
Éditorial
}

\section{Enfants en danger !}

(Conclusions de différentes études statistiques surtout valables pour les pays développés, mais les autres ne sont pas épargnés, ils sont soumis à d'autres dangers)

Si tu penses à 1 an, plante une graine, si tu penses à 10 ans, plante un arbre, si tu penses à 100 ans, change la conscience de l'Homme.

Proverbe Chinois

Dourquoi ce titre inquiétant alors que la médecine fait des progrès? Considérons quelques éléments de réflexion: on constate la présence de 100000 produits chimiques dans notre environnement proche depuis 1950, auxquels s'ajoutent 1000 nouveaux produits par an... Pour leur grande majorité, ces produits ne sont pas testés par rapport à la santé humaine... Et les tests existants sont effectués par les industriels eux-mêmes...

Ces produits ont souvent une influence hormonale, ce sont des perturbateurs endocriniens, et la notion ancienne de dose dangereuse n'est plus cohérente : on disait « la dose fait le poison » mais ces produits sont actifs même à des doses infimes, et quand ils sont associés on ne sait pas ce qu'il en advient (effet cocktail)...

Ces produits sont persistants : même interdits maintenant, les $\mathrm{PCB}$ (polychlorobiphényles cancérigènes et perturbateurs de la reproduction), le DDT, le mercure, etc. sont présents partout, y compris dans nos fleuves, aliments, et nos organismes...

Les « lobbies » alimentaires et pharmaceutiques sont ceux qui dépensent le plus en « lobbying » auprès des états et des politiques, et qui infiltrent efficacement les lieux de décision.

Une étude de 2010 montre que le corps d'un enfant de 10 ans contient en moyenne 128 résidus chimiques... 
Fœtus en danger dans le ventre de leur mère : les pollutions diverses, que nous allons évoquer plus loin, peuvent provoquer chez le fœtus des troubles pulmonaires et cardiaques, des fausses couches ou naissances prématurées, des perturbations sexuelles dues au dérèglement hormonal, une diminution du poids à la naissance, une diminution des défenses immunitaires, des troubles neurologiques et comportementaux, et sans doute d'autres pathologies. Et si la mère est inconsciente, elle peut ajouter un peu de fumée de tabac, d’alcool et de drogues diverses...

Bébés et enfants en danger avec leur nourriture : le lait maternel est le plus souvent pollué, car l'alimentation de la mère l'est également (dioxine, $P C B$, aluminium, arsenic, cadmium, mercure), les aliments provenant le plus souvent d'animaux (mammifères et poissons) nourris avec les résidus animaux et traités préventivement par des antibiotiques, et des végétaux traités...

Enfants en danger dans la maison : les composants nocifs sont principalement les dérivés pétroliers, les phtalates (dans les plastiques), les phénols et phyto-œstrogènes (polluants organiques persistants : POP), les métaux lourds qui sont stockés dans les cellules adipeuses et que l'on retrouve dans les analyses d'urine d'enfants de 8 ans. Ils se trouvent dans les différents produits de nettoyage, dans les sprays, les désinfectants, les peintures et vernis, les lessives, les colles, les substances ignifuges (brome dans les textiles et appareils ménagers), les shampoings, les insecticides, les désodorisants (même aux essences de plantes), les meubles en aggloméré (formol), les divers plastiques (emballages, contenants, jouets), les vêtements, l'alimentation, l'eau (les stations d'épuration non seulement ne les éliminent pas mais réactivent les résidus hormonaux), etc.

On retrouve encore les risques d'allergie, d'asthme, de dérèglement hormonal, de puberté précoce, de diabète, de cancer du sein, de la prostate, de diminution de la fertilité, d'asthme, d'allergies...

Rappelons aussi les violences familiales : 2 enfants meurent par jour en France.

Enfants en danger en dehors de la maison : les composants nocifs sont principalement l'ozone, le dioxyde d'azote et de soufre, et les particules ; ils sont principalement produits par les automobiles. Ces produits sont néfastes pour tous, mais encore plus pour les enfants, qui respirent à une fréquence plus grande que les adultes, et dont les tissus sont en formation et plus fragiles.

Ces substances irritent les bronches, perturbent ou détruisent les cellules ciliaires qui nettoient l'appareil respiratoire même si les enfants paraissent en bonne santé, et favorisent les allergies, les maladies cardio-vasculaires, les cancers du poumon et peut-être l'obésité.

Les poussettes des bébés sont à hauteur des tuyaux d'échappement (préférer les poussettes « kangourou » dans lesquelles les enfants sont surélevés, mais ce n'est évidemment pas miraculeux). Tout ceci, même si la pollution atmosphérique est dans les normes de l'OMS !

Il est conseillé de les sortir en dehors des heures d'affluence... mais quand ils sont amenés ou recherchés à la crêche ou à l'école, ce sont les heures d'affluence automobile...

La pollution étant souvent plus forte par beau temps, il faudrait les garder à la maison quand il fait beau... Pour les protéger du soleil, on les badigeonne d'anti-solaire avec d'autres substances nocives pour la plupart.

Et si les parents sont inconscients, ils ajoutent un peu de fumée de tabac. 
Évoquons également les risques pour leur colonne vertébrale et leur posture, que les sacs scolaires perturbent à cause de leur poids qui dépasse les normes admises par tous les spécialistes. Ceci est bien connu depuis très longtemps, mais aucune décision n'a encore été prise...

Évoquons enfin les abus de vaccinations multiples et leurs adjuvants, en particulier le mercure et l'aluminium, non dénués de toxicité.

Enfants en danger dans l'avenir : tous les dangers précédents qui vont persister de très nombreuses années vont être augmentés par de nouveaux dangers, l'électro-magnétisme de plus en plus fort, les nanoparticules (très invasives et en début d'utilisation sans qu'on en ait évalué les risques, elles sont prévues dans l'alimentation en 2015), les OGM (en plus de leurs inconvénients éventuels, les végétaux OGM, nécessitent progressivement des pesticides plus puissants car les parasites s'adaptent), les nouvelles pathologies liées au réchauffement climatique, les résistances aux antibiotiques qui augmentent considérablement, les vaccinations excessives et hasardeuses et l'industrie du tabac qui les attend avec impatience...

Ils (nous) mangent des particules de plastique dans les produits de la mer (250 milliards de particules en Méditerranée et 300 microparticules pour 300 grammes de chair de moule), dans la bière, le miel, etc. sans savoir les conséquences, sachant cependant déjà qu'ils contiennent des phalates, bisphénol et brome...

Questions à se poser : ne faut-il pas réfléchir et faire les études nécessaires avant de disperser dans la nature des substances ou technologies qui vont agir sur l'être humain et ne lui sont pas indispensables?

Est-ce que manger « normal », sain, n'est plus la normalité mais qu'il faille manger bio ! Et que manger sain devienne un luxe?

Après une période pendant laquelle les progrès techniques étaient considérés comme toujours bénéfiques pour l'être humain, ne faut-il pas maintenant se montrer plus lucides et circonspects ?

Est-il raisonnable d'utiliser des matériaux nocifs que l'on n'élimine pas après usage (plastiques, déchets nucléaires) et que l'on transmet généreusement aux générations futures ?

Peut-on promettre aux enfants de rester en vie plus longtemps ? Peut-être mais est-ce souhaitable si cela s'accompagne de maladies chroniques? On doit maintenant raisonner sur l'espérance de vie sans incapacité.

Quelles sont les conséquences économiques? Est-ce que traiter des maladies en s'intéressant plus aux symptômes et aux effets qu'aux causes dues à la pollution est le meilleur choix, si ce n'est pour les multinationales et leurs dirigeants, et est-ce du bon sens ( $89 \%$ du budget SS pour les traitements, $11 \%$ pour la prévention)?

Est-ce sain que les recherches pharmaceutiques soient financées, directement ou indirectement, par des intérêts privés qui ont pour objectif de faire des bénéfices (le « préventif » n'est pas rentable, alors que le « curatif » l'est) et non par les Universités qui auraient pour objectif la santé des citoyens? La plupart des scientifiques deviennent ainsi les « obligés » des industries.

\section{Sources d'optimisme relatif (enfin!)}

Les consommateurs deviennent plus informés et s'intéressent plus aux facteurs de bonne santé, ils devraient avoir de plus en plus d'influence sur les achats et donc sur les industriels. 
Les connaissances et découvertes scientifiques ne sont heureusement pas toutes négatives et feront prendre conscience de ces dangers, mais elles mettent du temps à être appliquées dans la vie courante et à être admises par les politiques car elles s'opposent souvent aux énormes intérêts financiers.

Les analyses deviennent plus précises et permettent de mieux détecter les polluants, globalement ils ont tendance à diminuer depuis 2000, mais les nouveaux sont plus difficiles à mettre en évidence...

\section{Conclusions}

II ne faut pas être bêtement contre le progrès, ni utiliser excessivement le principe de précaution, mais prendre le temps d'étudier les conséquences sur les organismes vivants de ce que nous diffusons dans l'environnement et la nourriture. La voie du juste milieu est presque toujours la meilleure, ainsi que la notion de " prendre le temps », nos enfants nous en remercieront.

Alain Benauwt

\section{Ouvrages à consulter}

Enfants en danger d'Anne Corinne Zimmer, Ed L'atelier

La coupe est pleine (nos enfants plus précieux que le CAC 40) de Sylvie Simon et Claire Séverac, Ed Elie \& Maso Un empoisonnement universel de Fabrice Nicolino, Ed Les liens qui libèrent Sang pour Sang toxique de JF. Narbonne, Ed Thierry Souccar

http://www.thierrysouccar.com/sante/info/pr-narbonne-la-dangerosite-des-polluants-actuels-est-difficilecerner-2333

Les opinions émises n'engagent que leurs auteurs. 